\title{
Nlrp3 activation in the intestinal epithelium protects against a mucosal pathogen
}

\author{
GX Song-Zhao ${ }^{1,4}, \mathrm{~N}$ Srinivasan ${ }^{1,4}, \mathrm{~J} \mathrm{Pott}^{1}, \mathrm{D}$ Baban $^{2}, \mathrm{G} \mathrm{Frankel}^{3}$ and KJ Maloy ${ }^{1}$
}

Polymorphisms in the intracellular pattern recognition receptor gene NLRP3 (NLR family, pyrin domain containing 3) have been associated with susceptibility to Crohn's disease, a type of inflammatory bowel disease. Following tissue damage or infection, NLRP3 triggers the formation of inflammasomes, containing NLRP3, ASC (apoptosis-associated speck-like protein containing a CARD domain), and caspase-1, that mediate secretion of interleukin (IL)-1 $\beta$ and IL-18. However, the precise role of NLRP3 inflammasomes in mucosal inflammation and barrier protection remains unclear. Here we show that upon infection with the attaching/effacing intestinal pathogen Citrobacter rodentium, NIrp3 ${ }^{-1-}$ and $A_{s c^{-1-}}$ mice displayed increased bacterial colonization and dispersion, more severe weight loss, and exacerbated intestinal inflammation. Analyses of irradiation bone marrow chimeras revealed that protection from disease was mediated through NIrp3 activation in nonhematopoietic cells and was initiated very early after infection. Thus, early activation of NIrp3 in intestinal epithelial cells limits pathogen colonization and prevents subsequent pathology, potentially providing a functional link between NLRP3 polymorphisms and susceptibility to inflammatory bowel disease.

\section{INTRODUCTION}

NOD-like receptors (NLRs) are cytosolic pattern recognition receptors that detect a variety of pathogen-associated molecular patterns as well as danger-associated molecular patterns and signal to coordinate multiple downstream pathways. ${ }^{1}$ When a subset of NLRs are triggered, they oligomerize with an adaptor protein known as ASC (apoptosis-associated speck-like protein containing a CARD domain), and a proenzyme, caspase-1, to form a multimeric protein complex known as the inflammasome. ${ }^{1}$ Inflammasomes activate caspase- 1 that leads to the processing and secretion of the inflammatory cytokines interleukin (IL)-1 $\beta$ and IL-18 and to a form of inflammatory cell death termed pyroptosis. ${ }^{1-3}$ Recently, single-nucleotide polymorphisms in the regulatory region of NLRP3 (NLR family, pyrin domain containing 3), resulting in lowered expression of the inflammasome-forming NLRP3, were linked with susceptibility to Crohn's disease and this was attributed to decreased expression of IL-1 $\beta$ from monocytes. ${ }^{4}$ Moreover, several recent studies in mice reported that Nlrp3 inflammasome activation contributed to protection from chemically induced intestinal inflammation through secretion of IL-18, ${ }^{5-7}$ although other investigators reported conflicting results. ${ }^{8-11}$

To investigate the role of the Nlrp3 inflammasome in infection-associated intestinal inflammation, we employed Citrobacter rodentium, an attaching and effacing intestinal pathogen that is a model of enteropathogenic Escherichia coli infections in humans. ${ }^{12,13}$ In order to colonize the intestinal mucosa, $C$. rodentium uses a type III secretion system (T3SS) to deliver effector proteins that allows attachment to epithelial cells and subversion of host signaling pathways, triggering cytoskeletal rearrangements. This results in the formation of hallmark attaching/effacing lesions on the apical epithelium, characterized by intimate bacterial attachment and effacement of the brush border microvilli. ${ }^{12,13}$ How the innate immune system detects these virulent assaults to the epithelium and mounts an antimicrobial response toward C. rodentium is poorly characterized. In this study, we show that very early during the course of the infection, activation of Nlrp3 and Asc within nonhematopoietic cells serves to limit tissue bacterial burdens and consequently dampens intestinal inflammation.

\footnotetext{
${ }^{1}$ Sir William Dunn School of Pathology, University of Oxford, Oxford, UK. ${ }^{2}$ Wellcome Trust Centre for Human Genetics, University of Oxford, Oxford, UK and ${ }^{3}$ MRC Centre for Molecular Bacteriology and Infection, Division of Cell and Molecular Biology, Imperial College London, London, UK. Correspondence: KJ Maloy (kevin.maloy@path.ox.ac.uk)

${ }^{4}$ These authors contributed equally to this work.
} 


\section{RESULTS}

$\mathrm{NIrp3}^{-/-}$and $\mathrm{Asc}^{-/-}$mice display exacerbated intestinal inflammation upon $C$. rodentium infection

To investigate the role of the Nlrp3 inflammasome in bacterially triggered intestinal inflammation, we infected cohorts of wild-type (WT), Nlrp3 ${ }^{-/-}$, and $\mathrm{Asc}^{-/-}$mice with $C$. rodentium, a mouse intestinal pathogen that causes transient diarrhea and intestinal inflammation. ${ }^{12,13}$ It was recently reported that mice lacking a related inflammasomeforming NLR, Nlrp6 $6^{-/-}$mice, as well as Casp1 $1^{-/-}$and $A s c^{-/-}$ mice, harbored a more "colitogenic" intestinal microbiota that exacerbated acute intestinal inflammation induced by dextran sulfate sodium (DSS) administration. ${ }^{14}$ This "colitogenic" flora and susceptibility phenotype could be transferred to WT mice by co-housing before DSS challenge. ${ }^{14}$ Therefore, to circumvent the microbiota as a potential contributing factor to any observed differences in $N \operatorname{lrp3} 3^{-/-}$and $A s c^{-/-}$mice, these mice were co-housed with WT mice for at least 2 weeks before infection with $C$. rodentium. Following infection with $C$. rodentium, mice were either resegregated by genotype, or were co-housed for the entire period of infection. We observed identical results irrespective of the overall period of co-housing.

Following oral infection with $C$. rodentium, WT mice maintained their original body weight throughout the course of infection, whereas $N l r p 3^{-/-}$and $A s c^{-/-}$mice lost significant amounts of weight between day 7 and day 14 after infection, before returning to their original weight by day 21 (Figure 1a). Interestingly, $\mathrm{Nlrp3}^{-/-}$mice lost less weight than $\mathrm{Asc} \mathrm{C}^{-/-}$ mice after infection, suggesting the involvement of other inflammasome-forming NLRs in protective immunity against C. rodentium or inflammasome-independent, Asc-driven functions. Overall, these results indicated that signaling through Nlrp3 and Asc protected against C. rodentiuminduced wasting disease.

Consistent with the absence of systemic disease, C. rodentium infection of WT mice led to only mild intestinal inflammation with limited colonic hyperplasia, and leukocyte infiltration (Figure 1b-d). In contrast, Nlrp3 $3^{-/-}$and $A s c^{-/-}$mice developed severe typhlitis and colitis after infection with C. rodentium, characterized by crypt loss, submucosal inflammation, prominent edema, leukocyte infiltration, and increased expression of inflammatory cytokines (Figure $\mathbf{1 b}$-d and Supplementary Figure S1 online). Thus, activation of Nlrp3 and Asc limits C. rodentium-driven intestinal inflammation.

\section{NIrp3 and Asc activation limits $C$. rodentium colonization and systemic translocation}

To determine whether the exacerbated intestinal inflammation in $N$ lrp3 $3^{-/-}$and $A s c^{-/-}$mice was because of an inability to control bacterial burdens, we measured C. rodentium levels at

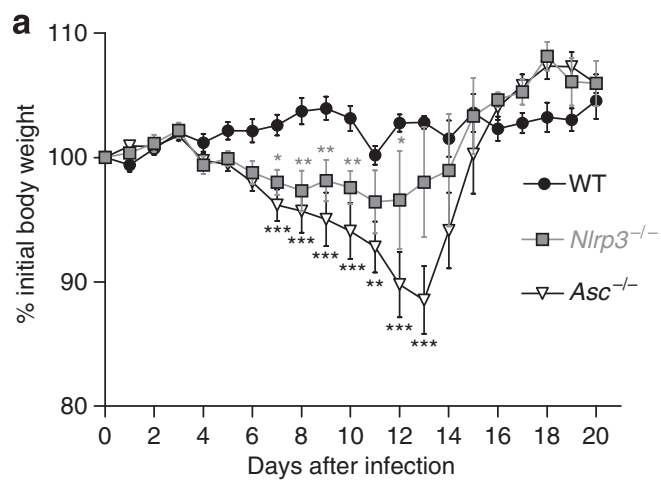

b
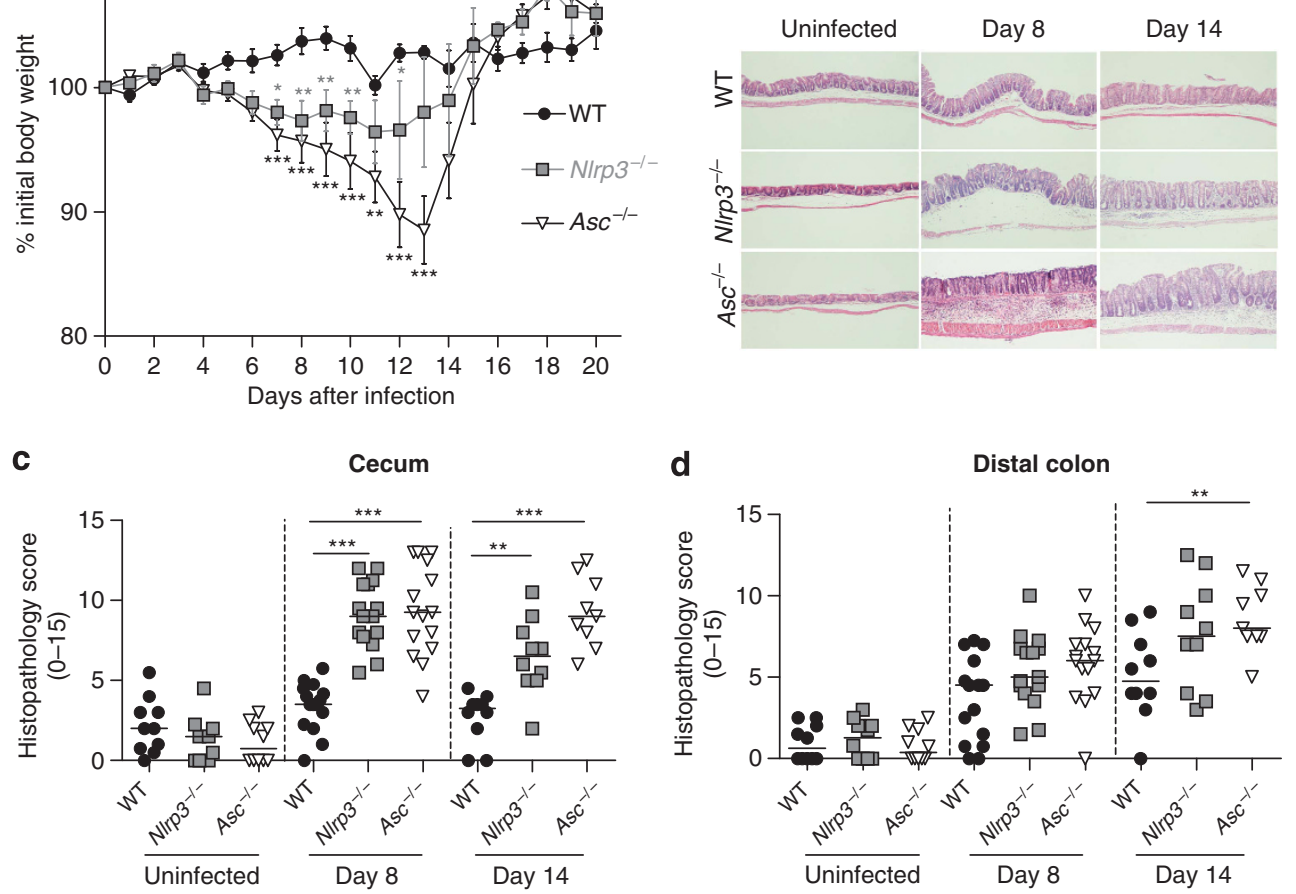

Figure 1 Nlrp3 and Asc activation protects against Citrobacter rodentium-induced wasting disease and intestinal inflammation. Wild-type (WT), NIrp3 ${ }^{-1-}$, and $A s C^{-1-}$ mice were infected orally with $\sim 10^{9} \mathrm{C}$. rodentium. Cohorts were killed 8 and 14 days after infection and assessed for intestinal inflammation. (a) Body weights of WT, NIrp3 ${ }^{-1-}$, and $\mathrm{Asc}^{-1-}$ mice. Symbols denote mean weights ( \pm s.e.m.) as a percentage of the initial body weight $(n=4-16)$. (b) Representative photomicrographs depicting hematoxylin and eosin (H\&E) staining of $C$. rodentium-infected ceca (original magnification $\times 50$ ). Inflammation scores in the (c) cecum and (d) distal colon were assessed as described in the Methods $(n=9-15)$. Data represent either a representative experiment (one of three independent experiments (a)) or pooled results from two to three independent experiments $(\mathbf{c}, \mathbf{d})$. Horizontal bars represent the median and each symbol represents an individual mouse. Statistical significance was determined by either the two-way analysis of variance (ANOVA) (a) or the Mann-Whitney test $(\mathbf{c}, \mathbf{d})\left({ }^{\star} P<0.05 ;{ }^{\star \star} P<0.01 ;{ }^{* \star \star} P<0.001\right)$. 
the site of infection. Fitting with exacerbated intestinal inflammation, we observed significantly higher bacterial burdens in the cecum and distal colon of $\mathrm{Nrp3}^{-/-}$and $\mathrm{Asc}^{-/-}$mice as compared with WT mice on both days 8 and 14 after infection (Figure 2a,b). To examine whether Nlrp3 activation limited $C$. rodentium attachment to the epithelium, we visualized the infection by immunofluorescence. We observed that whereas $C$. rodentium was mainly localized to the luminal surface of the epithelium in WT mice, the bacterium was able to penetrate deep into the base of the crypts in $A s c^{-/-}$and $N l r p 3^{-/-}$mice (Figure 2c).
This indicated that activation of Nlrp3 and Asc limited bacterial burdens and restricted their localization within the intestine.

C. rodentium is thought to cause diarrhea partly by weakening the tight junctions between epithelial cells. ${ }^{15}$ One potential consequence of this breach is translocation of bacteria past the intestinal barrier into systemic sites. To determine the degree of this translocation we measured the $C$. rodentium levels in the spleen. Strikingly, although we observed no translocation of C. rodentium in WT spleens, $80 \%$ of $A s c^{-/-}$mice and $50 \%$ of $N \mathrm{~Np}^{-/-}$mice harbored detectable numbers of C. rodentium a

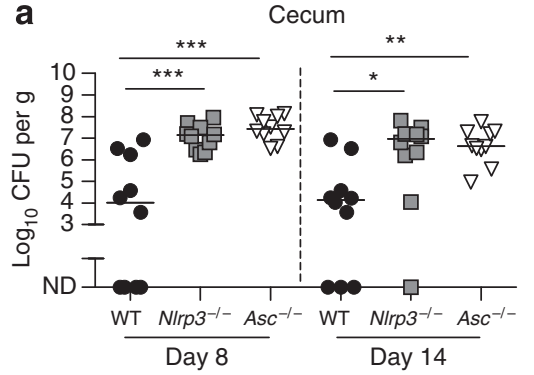

C
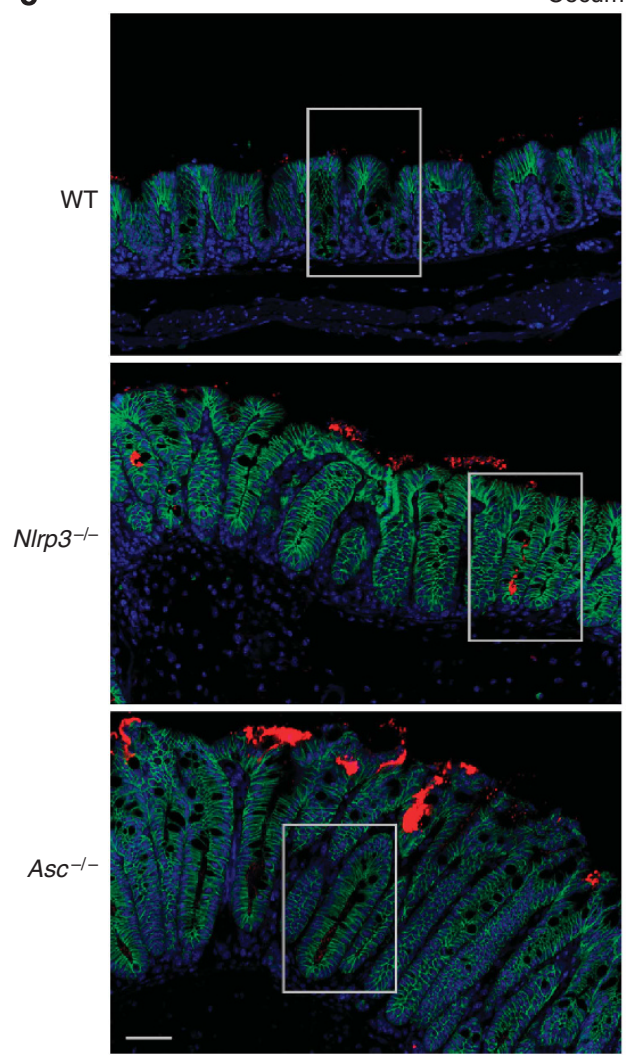

b
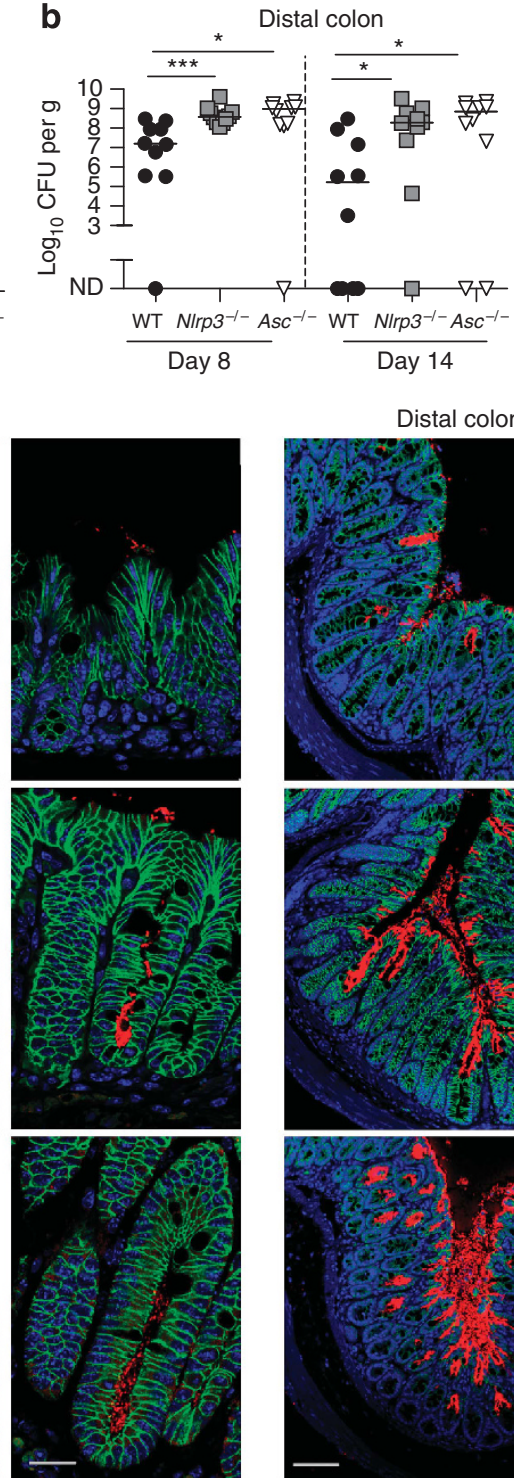

Distal colon
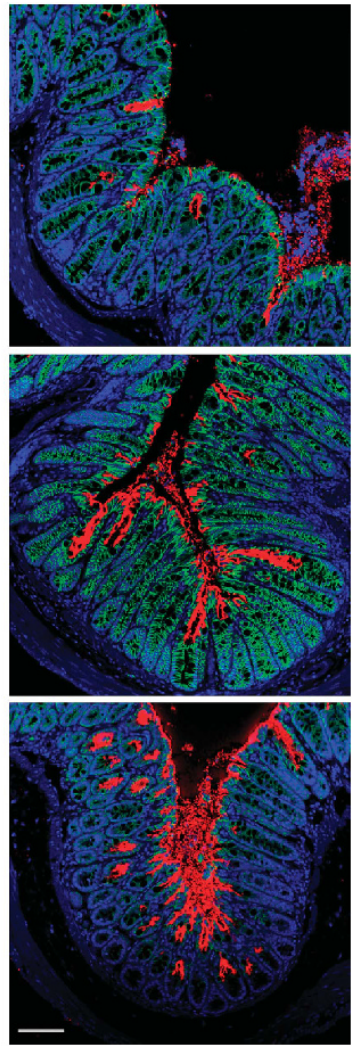

C. rodentium E-cadherin DAPI

Day 8 after infection

Figure 2 Activation of the NIrp3 and Asc limits Citrobacter rodentium burdens. Wild-type (WT), NIrp3 ${ }^{-/-}$, and Asc ${ }^{-/-}$mice were infected orally with $\sim 10^{9}$ C. rodentium. Cohorts were killed at 8 and 14 days after infection. $C$. rodentium burdens were measured in the (a) cecum and (b) distal colon as described in the Methods (ND, no detectable bacteria). (c) Ceca from infected WT, NIrp3 ${ }^{-1-}$, and $A s C^{-/-}$mice (8 days after infection) were stained for $C$. rodentium (red), E-cadherin (green), and 4',6-diamidino-2-phenylindole (DAPI; blue). Left panel, bar $=30 \mu \mathrm{m}$; mid panel, bar $=15 \mu \mathrm{m}$; and right panel, bar $=70 \mu \mathrm{m}$. Data represent pooled results from at least two independent experiments $(\mathbf{a}, \mathbf{b})$. Horizontal bars represent the medians and each symbol represents an individual mouse $(n=10)$. Statistical significance was determined by the Mann-Whitney test. $\left({ }^{\star} P<0.05 ;{ }^{\star \star} P<0.01 ;{ }^{\star \star \star} P<0.001\right)$. 
in their spleens at 8 days after infection (Figure 3a). In accordance with the presence of $C$. rodentium in the spleen, we also observed significant splenomegaly in $N l r p 3^{-/-}$ and $A s c^{-/-}$mice, but not in WT mice (Figure 3b). Splenomegaly was associated with greater accumulation of granulocytes $\left(\mathrm{CD} 11 \mathrm{~b}^{+} \mathrm{GR} 1^{\mathrm{hi}}\right)$ in $\mathrm{Nlrp3}^{-/-}$and $\mathrm{Asc}^{-/-}$ spleens compared with WT spleens (Figure 3c,d). These results indicate that signaling via Nlrp3 and Asc is important for limiting systemic translocation of $C$. rodentium, and for preventing excessive systemic inflammatory responses.

\section{Caspase-1 activation is intact in the intestines of C. rodentium-infected $\mathrm{NIrp3}^{-/-}$mice}

Triggering of the Nlrp3 inflammasome in leukocytes results in the activation of caspase- 1 and subsequent maturation and secretion of the cytokines IL- $1 \beta$, IL- $1 \alpha$, and IL- $18 .{ }^{16,17}$ However, we observed no induction of IL- $1 \beta$ during the course of infection, nor any differences in the relative IL-1 $\beta$ levels between WT and $A s c^{-/-}$or Nlrp3 $3^{-/-}$mice before or during infection (Figure 4a). Similarly, levels of IL- $1 \alpha$ were also very low and we found no difference between WT and $A s c^{-/-}$or Nlrp3 $3^{-1-}$ mice (Figure 4a). In contrast, we observed almost no IL-18 production by intestinal explants from $A s c^{-/-}$mice throughout the course of infection; however, Nlrp3 $3^{-1-}$ mice produced slightly higher levels of IL-18 than WT mice (Figure 4a). Fitting with this pattern of IL-18 production, we found equal or greater levels of active caspase-1 (p10) and mature IL-18 in the intestines of $N l r p 3^{-/-}$mice as compared with WT mice at 8 days after infection, whereas caspase- 1 p10 and mature IL-18 were barely detectable in $A s c^{-/-}$mice (Figure 4b). Importantly, this result suggests that the susceptibility phenotype observed in $N l r p 3^{-1-}$ mice may be caspase- 1 independent. IL-18 has been implicated in protecting the intestinal epithelium from DSS-induced colitis; ${ }^{5-7}$ thus, defective IL-18 production in $A s c^{-/-}$mice may explain the slightly exacerbated disease phenotype observed in $A s c^{-/-}$ mice relative to Nlrp3 $3^{-/-}$mice. However, as IL-18 production was intact in $N l r p 3^{-/-}$mice, these results indicate that the major susceptibility phenotype of $N \operatorname{lrp3} 3^{-/-}$mice to C. rodentium cannot be attributed to a lack of IL-18 production. Consistent with a minor role for IL-18, when we compared Illsr $1^{-/-}$and WT mice after 8 days after infection, we found only a small trend toward moderate cecal inflammation in Ill $8 \mathrm{r}^{-/-}$mice that was not statistically significant (Figure 4c). However, IL-18 production did contribute to limiting bacterial burdens as $I l 18 r 1^{-/-}$mice harbored significantly higher C. rodentium levels in their ceca as compared with WT mice (Figure 4d). Furthermore, similar to $A s c^{-/-}$mice, we observed translocation of $C$. rodentium into the spleens in $\sim 80 \%$ of $1118 r 1^{-/-}$mice (Figure 4d). Collectively, these data suggest that IL-18 might explain the difference in disease severity between $N \operatorname{lrp} 3^{-/-}$and $A s c^{-/-}$mice. Signaling via NIrp3 and Asc in nonhematopoietic cells
limits bacterial colonization and intestinal inflammation The adaptive immune system, specifically $B$ cell-derived immunoglobulin $\mathrm{G}$ and Th17 cells, is crucial for protection against and clearance of $C$. rodentium. ${ }^{18-21}$ The eventual recovery of the $\mathrm{Nlrp}^{-/-}$and $A s \mathrm{c}^{-/-}$mice from C. rodentium suggested that essential adaptive clearance mechanisms were not significantly compromised and this was confirmed by analyses of effector Th17 cell responses at late stages of infection, which were equivalent in WT, $N \operatorname{lrp} 3^{-/-}$, and $A s c^{-/-}$mice (data not shown). To confirm that susceptibility of Nlrp3 $3^{-/-}$and $A s c^{-/-}$ mice to $C$. rodentium stemmed from an inability to mount an effective innate immune response, we generated Rag1 $^{-/-}$ $N \operatorname{Nrp3} 3^{-1-}$ and $R a g 1^{-/-} A s c^{-/-}$mice, lacking mature B and $\mathrm{T}$ cells, and infected them with $C$. rodentium. At 8 days after infection, we observed that $R a g 1^{-/-} N \operatorname{Nrp} 3^{-/-}$and $\mathrm{Ragl}^{-/-} \mathrm{Asc}^{-/-}$mice still displayed significantly higher levels of intestinal inflammation compared with $R a g 1^{-/-}$mice, indicating that Nlrp3 and Asc confer protection against C. rodentium independently of the adaptive immune response (Supplementary Figure S2).

To determine the cell types responsible for Nlrp3/Ascmediated protection, we generated irradiation bone marrow (BM) chimeras selectively lacking Nlrp3 or Asc in distinct cellular compartments (i.e., hematopoietic or intestinal tissue cells) and infected them with $C$. rodentium. In agreement with our previous observations, control $A s c^{-/-} \rightarrow A s c^{-/-}$and $N \operatorname{Nrp3} 3^{-/-} \rightarrow \mathrm{Nlrp3}^{-/-}$BM chimeric mice displayed enhanced intestinal inflammation and weight loss compared with WT $\rightarrow$ WT mice (Figure 5a,b). Selective restoration of Nlrp3 expression in the hematopoietic compartment (WT $\rightarrow$ $N \operatorname{lrp} 3^{-/-}$) conferred no significant protection from C. rodentium-induced wasting disease, whereas mice with selective expression of Nlrp3 in the nonhematopoietic compartment $\left(\mathrm{Nlrp3} 3^{-/-} \rightarrow \mathrm{WT}\right) \quad$ exhibited robust protection (Figure 5a). Similarly, although mice in which $A s c$ was selectively restored in the hematopoietic compartment $\left(\mathrm{WT} \rightarrow A s c^{-/-}\right)$exhibited an intermediate phenotype, selective expression of $A s c$ in the nonhematopoietic compartment $\left(A s c^{-/-} \rightarrow \mathrm{WT}\right)$ provided complete protection from weight loss (Figure 5b). An identical disease pattern was observed for intestinal pathology; mice with selective expression of $N \operatorname{lrp} 3\left(\mathrm{Nlrp3} 3^{-/} \rightarrow \mathrm{WT}\right)$ or Asc $\left(A s c^{-/-} \rightarrow \mathrm{WT}\right)$ in the nonhematopoietic compartment were protected from intestinal inflammation, whereas the reciprocal chimeras lacking $N l r p 3\left(\mathrm{WT} \rightarrow N l r p 3^{-/-}\right.$) or Asc (WT $\rightarrow$ $A s c^{-/-}$) in the nonhematopoietic compartment exhibited severe intestinal inflammation (Figure $\mathbf{5 c}, \mathbf{d}$ ). Consistent with a minor role for hematopoietic Asc expression, $A s c^{-/-} \rightarrow \mathrm{WT}$ mice had slightly more severe typhlitis than $\mathrm{WT} \rightarrow \mathrm{WT}$ mice, but significantly lower levels than $A s c^{-/-} \rightarrow A s c^{-/-}$mice at 14 days after infection (Figure 5d).

Earlier, we observed that the exacerbated phenotype in $A s c^{-/-}$ and Nlrp3 $3^{-1-}$ mice was accompanied by higher bacterial burdens at the site of infection (Figure 2a,b). Correspondingly, BM chimeras that selectively lacked Nlrp3 (WT $\rightarrow$ $N \operatorname{Nrp} 3^{-/-}$) or Asc (WT $\rightarrow A s c^{-/-}$) in the nonhematopoietic compartment also harbored significantly higher $C$. rodentium levels in the cecum and distal colon (Figure 5e,f), although there again appeared to be a minor role for Asc in hematopoietic cells 
a

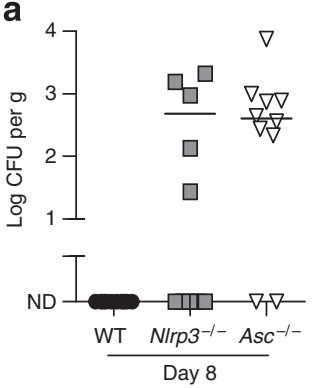

C
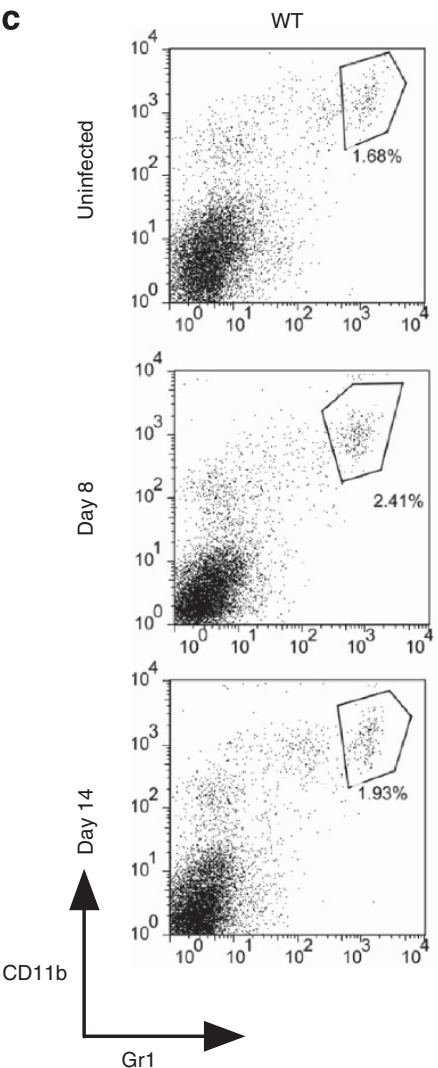

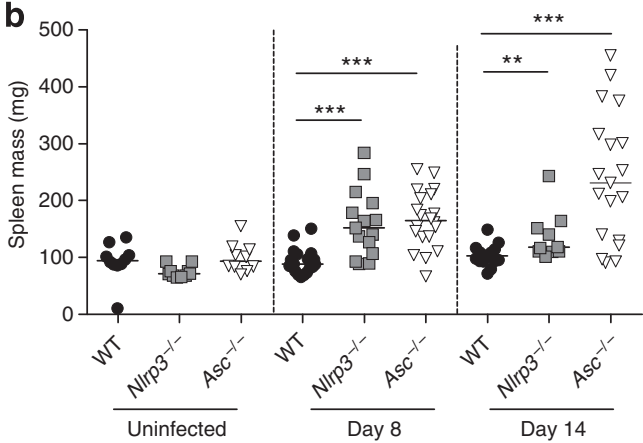

$A s c^{-1-}$
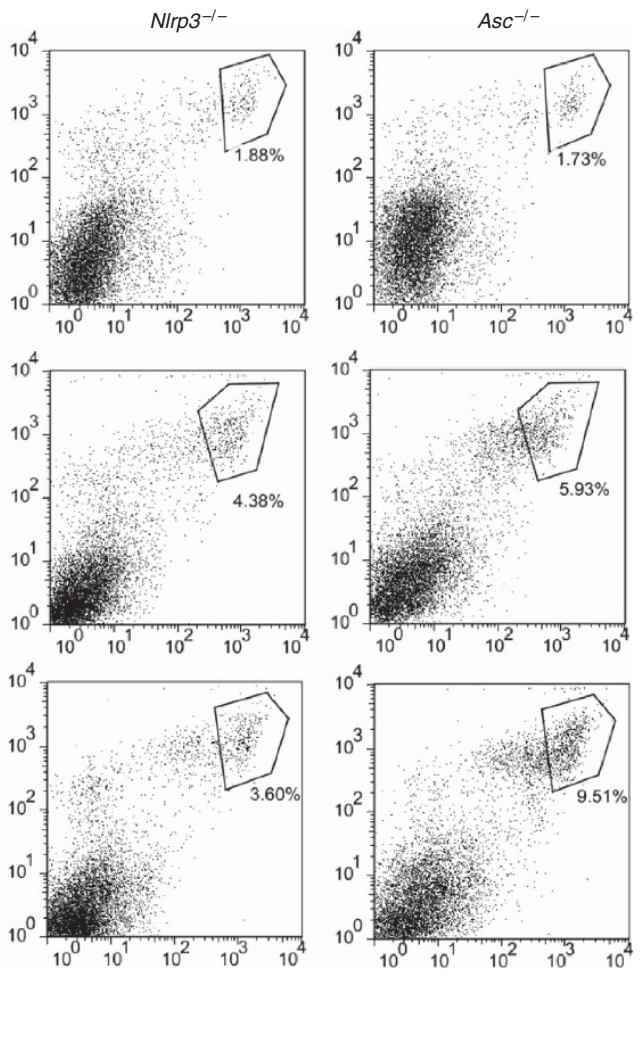

d

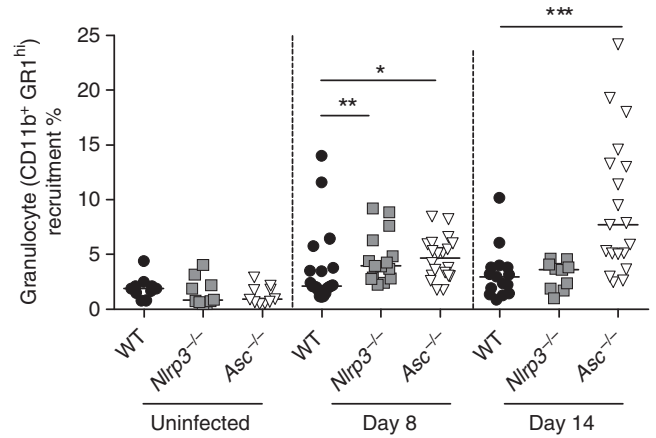

Figure 3 Citrobacter rodentium-infected $\mathrm{NIrp3}^{-/-}$and $\mathrm{Asc}^{-/-}$mice cannot restrict bacterial translocation and display systemic inflammation. Wild-type (WT), NIrp3 ${ }^{-1-}$, and $A s c^{-1-}$ mice were orally infected with $\sim 10^{9}$ C. rodentium and killed 8 or 14 days after infection. (a) Splenic bacterial loads at 8 days after infection. (b) Spleen weights. (c) Representative fluorescence-activated cell sorting (FACS) plots of splenic granulocytes (CD11b ${ }^{+}$ $\mathrm{Gr}^{\mathrm{hi}}$ ). (d) Frequency of granulocytes in the spleen (CD11b $\mathrm{Gr}^{\mathrm{hi}}$ ). Each symbol represents a single animal and data represent pooled results from at least two independent experiments $(n=9-20)$. Horizontal bars represent group medians. Statistical significance was determined by the Mann-Whitney test $\left({ }^{\star} P<0.05 ;{ }^{* *} P<0.01 ;{ }^{* * *} P<0.001\right)$. 


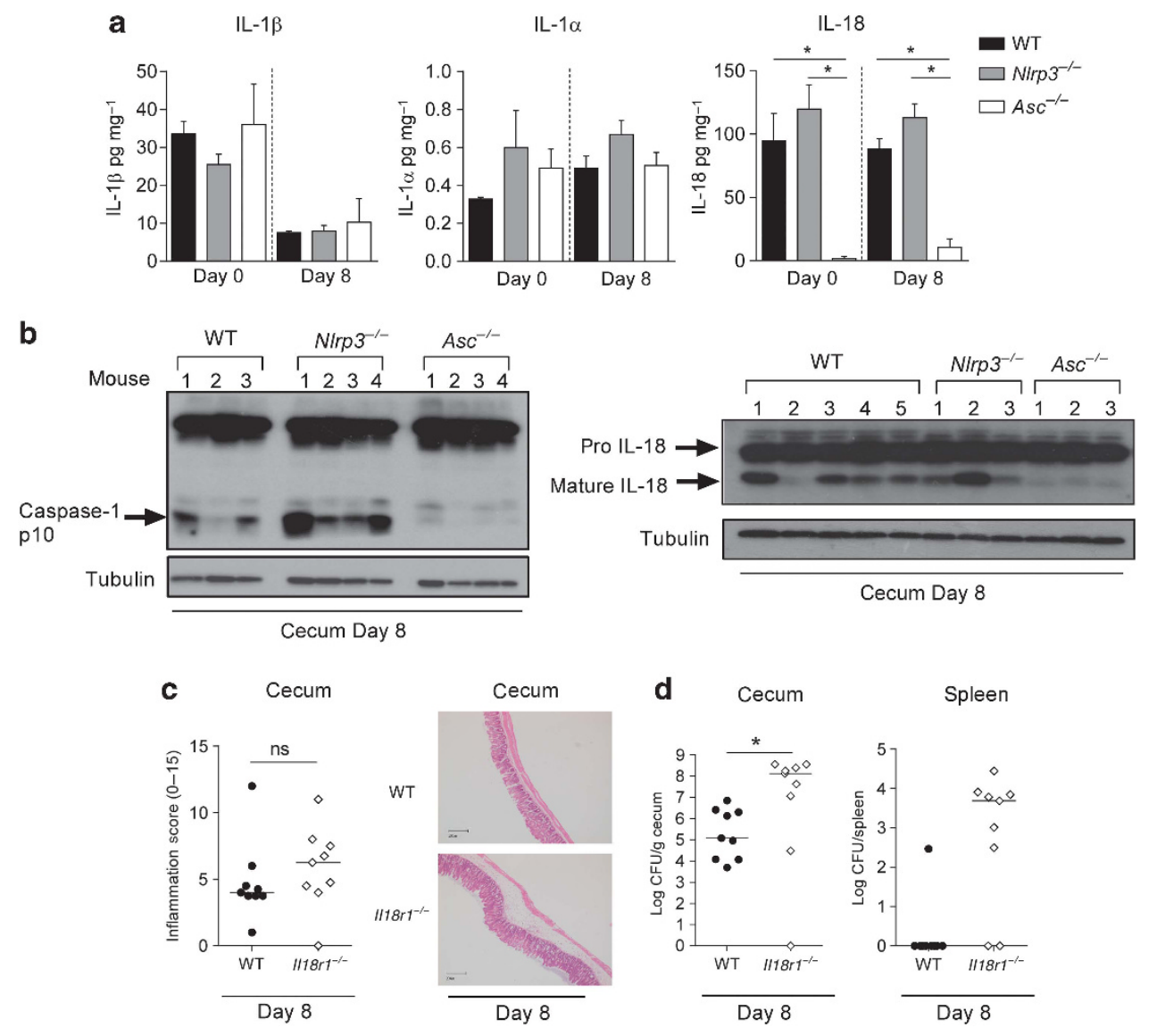

Figure 4 Caspase-1 activation is intact in the intestines of $N / r p 3^{-/-}$mice. Cohorts of wild-type (WT), NIrp3 $3^{-/-}$, II18r1 $1^{-/-}$and $A s c^{-/-}$mice were infected with $\sim 10^{9}$ Citrobacter rodentium and killed 8 days after infection. (a) Protein levels of interleukin (IL)-1 $\beta$, IL-1 $\alpha$, and IL-18 in the cecal organ explants $(n=4)$. (b) Immunoblot analysis of cecal protein extracts from WT, NIrp3 ${ }^{-1-}$, and Asc ${ }^{-1-}$ mice probed with antibody to caspase-1, IL-18, and tubulin $(n=3-5)$. (c) Inflammation scores in the cecum in WT and $/ 118 r^{-1}-$ mice $(n=9)$ and representative photomicrographs depicting hematoxylin and eosin (H\&E) staining of $C$. rodentium-infected ceca. (d) $C$. rodentium burdens were measured in the cecum and spleen in WT and $1 / 18 r 1^{-1-}$ mice $(n=9)$. Each symbol represents a single animal and is either representative of two experiments $(\mathbf{a}, \mathbf{b}, \mathbf{c})$ or pooled from two independent experiments (d). Bar graphs represent means ( \pm s.e.m.). Horizontal bars represent the median. Statistical significance was determined by the Mann-Whitney test $\left({ }^{*} P<0.05\right)$.

(Figure 5f). To determine whether Nlrp3- or Asc-mediated protection correlated with caspase-1 activation, we assessed caspase-1 cleavage. Intriguingly, caspase-1 activation (p10) was absent in the ceca of mice lacking Asc in nonhematopoietic cells $\left(\mathrm{WT} \rightarrow A s c^{-1-}\right)$ (Figure 5g), indicating that the majority of caspase-1 activation occurs in nonhematopoietic cells in the gut. However, caspase-1 activation was unimpaired in all the Nlrp3 chimera groups (Figure 5g), confirming our earlier conclusion that caspase-1 activation is still intact in $N \mathrm{Nrp3}^{-/-}$mice. Our earlier results suggested that Asc-dependent IL-18 production partially limited C. rodentium burdens in the cecum (Figure 4d). To assess the cellular compartment responsible for intestinal IL-18 production, we performed Western blotting in tissue samples from the BM chimeras. We observed that mature IL-18 expression in the intestine correlated strongly with Asc expression in nonhematopoietic cells as mature IL-18 expression was markedly reduced in $\mathrm{WT} \rightarrow A s c^{-/-} \mathrm{BM}$ chimeras, but remained intact in $\mathrm{Asc}^{-/-} \rightarrow \mathrm{WT}$ BM chimeras (Supplementary Figure S3). Consistent with a dominant protective role for nonhematopoietic Nlrp3 and Asc activation, immunofluorescence analysis indicated that Asc was strongly and ubiquitously expressed by E-cadherin ${ }^{+}$intestinal epithelial cells (IECs), whereas very few cells in the lamina propria had detectable Asc expression (Figure 5h). Taken together, our findings show that activation of Nlrp3-Asc signaling in the intestinal epithelium limits $C$. rodentium colonization and confers protection against intestinal inflammation.

\section{Nlrp3 and Asc activation mediates early control against C. rodentium}

We next assessed the mechanism through which Nlrp3 and Asc activation conferred protection against C. rodentium. Asc ${ }^{-1-}$ and $\mathrm{Nlrp3}^{-/-}$mice harbored higher C. rodentium loads compared with WT mice, and thus we hypothesized that impaired control of bacterial colonization might underlie the enhanced severity of intestinal inflammation in these mice. However, some enteric pathogens such as Salmonella gain a competitive advantage under inflammatory environments that allow them to outgrow the intestinal microbiota. ${ }^{22,23}$ Thus, to determine whether the outgrowth of C. rodentium in Nlrp $3^{-1-}$ and $\mathrm{Asc}^{-/-}$mice was the cause or the consequence of the severe intestinal inflammation, we focused on an early time point after 
a

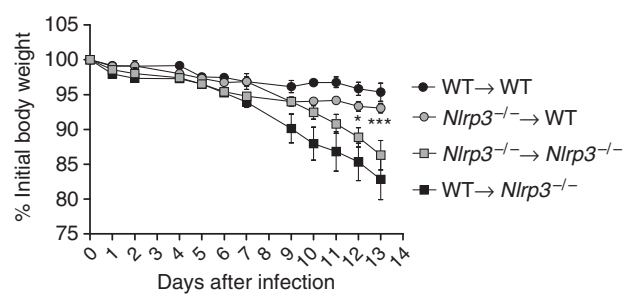

b

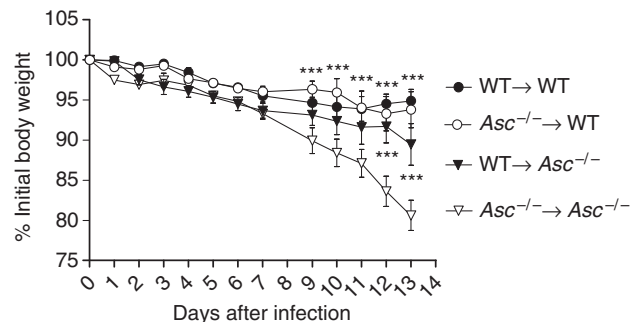

e

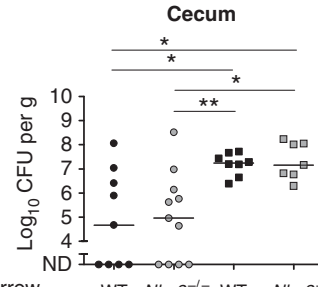

Bone marrow

WT $\mathrm{NIrp3}^{-1-} \mathrm{WT} \quad \mathrm{NIrp3}^{-1}$ Nonhematopoietic WT WT NIrp3-1- $\mathrm{NIrp3}^{-1-}$

$$
\text { Day } 8
$$

f

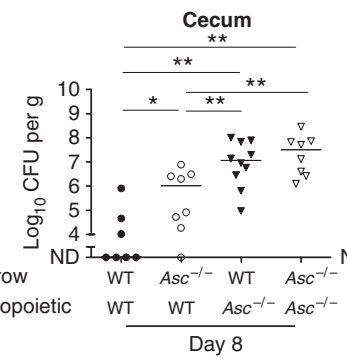

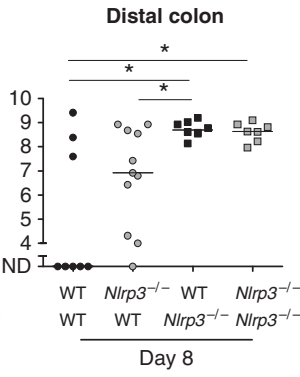

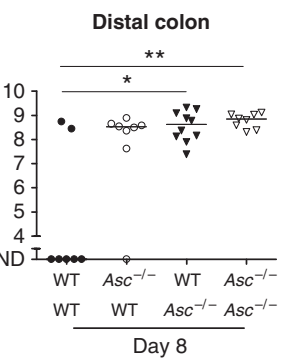

C

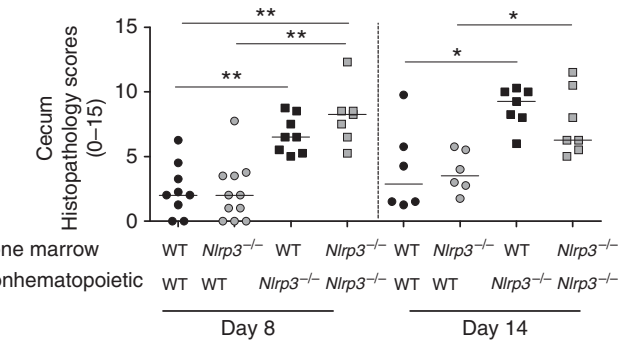

d

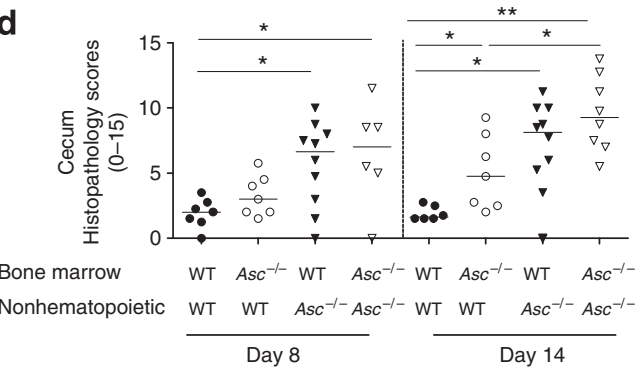

g

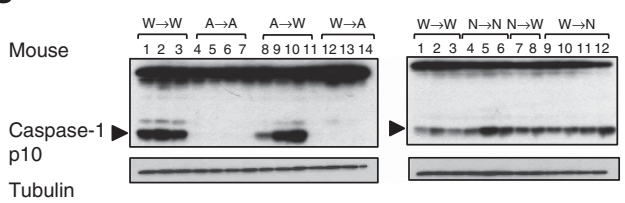

h
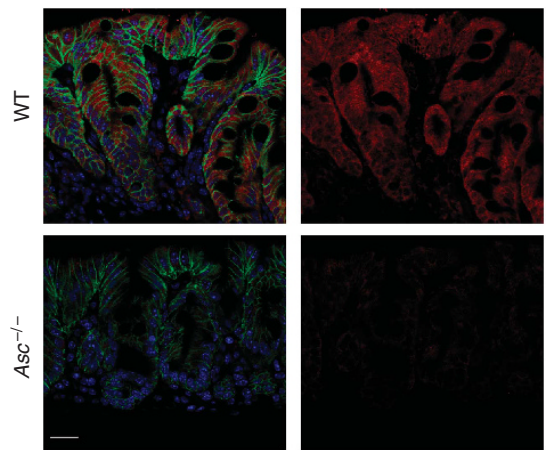

Asc E-cadherin DAPI

Figure 5 Nlrp3 and Asc signaling in nonhematopoietic cells provides protection from Citrobacter rodentium-induced intestinal inflammation. Irradiation bone marrow chimeras were generated as described in the Methods and infected with $\sim 10^{9} \mathrm{C}$. rodentium and killed at 8 or 14 days after infection. Body weights of (a) NIrp3 $3^{-1-}$ and (b) $A s c^{-\prime-}$ bone marrow chimeras. Symbols denote mean weights ( \pm s.e.m.) as a percentage of the initial body weight. Statistical significance was determined between chimera groups and controls, i.e., $A s c^{-/} \rightarrow A s c^{-/-}$ and $\mathrm{NIrp3}^{-I_{-}} \rightarrow \mathrm{NIrp3}^{-/-}$, respectively. Inflammation scores in the cecum of (c) NIrp3 ${ }^{-/-}$and (d) $A s C^{-/-}$bone marrow chimeras. C. rodentium loads in the cecum and distal colon of (e) NIrp3 ${ }^{-/-}$and (f) $A s c^{-1-}$ bone marrow chimeras at 8 days after infection (ND, no detectable bacteria). (g) Immunoblot analysis of total cecal protein extracts from wild-type (WT), NIrp3 ${ }^{-1-}$, and $A s C^{-1-}$ chimeras at 8 days after infection probed with antibody to caspase-1 and tubulin ( $n=2-4)$. (h) Ceca from WT and Asc $^{-1-}$ mice were stained for Asc (red), E-cadherin (green), and 4',6-diamidino-2-phenylindole (DAPI; blue). Bar $=15 \mu \mathrm{m}$. Data represent pooled results from two to three independent experiments, and each symbol represents a single mouse with at least six mice per group. Horizontal bars represent the median. Statistical significance was determined by either (a, b) the two-way analysis of variance (ANOVA) or $(\mathbf{c}-\mathbf{f})$ the Mann-Whitney test $\left({ }^{\star} P<0.05 ;{ }^{* *} P<0.01 ;{ }^{* * *} P<0.001\right)$.

infection. Strikingly, we found that at just $72 \mathrm{~h}$ after infection, $N \operatorname{Np} 3^{-1-}$ and $A s c^{-1-}$ mice already harbored $\sim 1,000$-fold more tissue-adherent $C$. rodentium in their ceca than WT mice (Figure 6a), with clear evidence of penetration into the crypts (Figure 6b), even though no intestinal pathology was detectable at this early time point (Figure 6c,d). Thus, Nlrp3 and Asc activation is triggered very early in response to $C$. rodentium and functions to limit bacterial colonization and subsequent disease.
Recent studies have identified a crucial role for IL-22producing $\mathrm{ROR}_{\mathrm{r}} \mathrm{t}^{+}$innate lymphoid cells in early innate immunity against $C$. rodentium. ${ }^{24,25}$ IL-22 mediates protection by inducing the secretion of antimicrobial peptides (AMPs) such as RegIII $\gamma$ by IECs. ${ }^{26}$ However, we observed similar frequencies of innate lymphoid cells (Figure 6e) and efficient induction of $I l 22, \operatorname{Reg} 3 g$, and $I l 17 a$ in the ceca of WT, $N l r p 3^{-/-}$, and $A s c^{-/-}$mice at $72 \mathrm{~h}$ after infection 
a

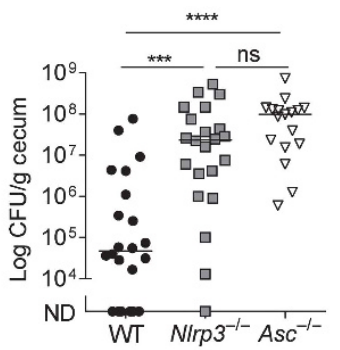

d

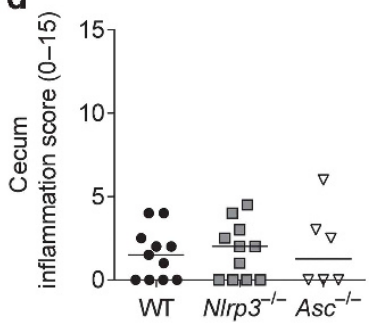

b
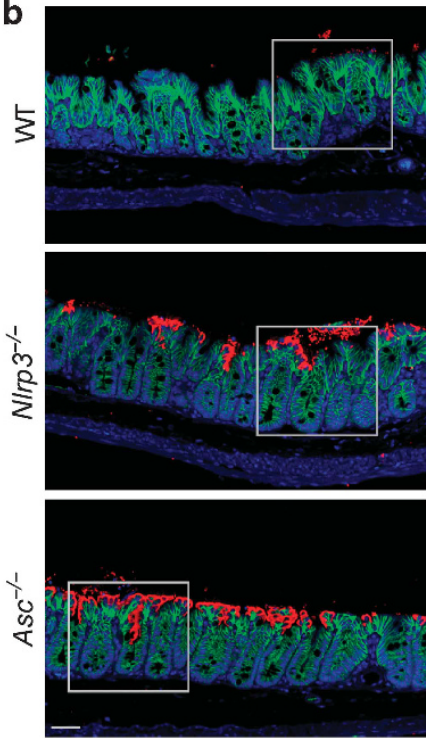

C. rodentium E-cadherin DAPI
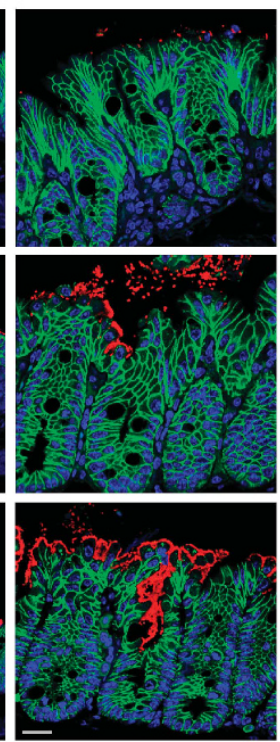

$\operatorname{Reg} 3 g$
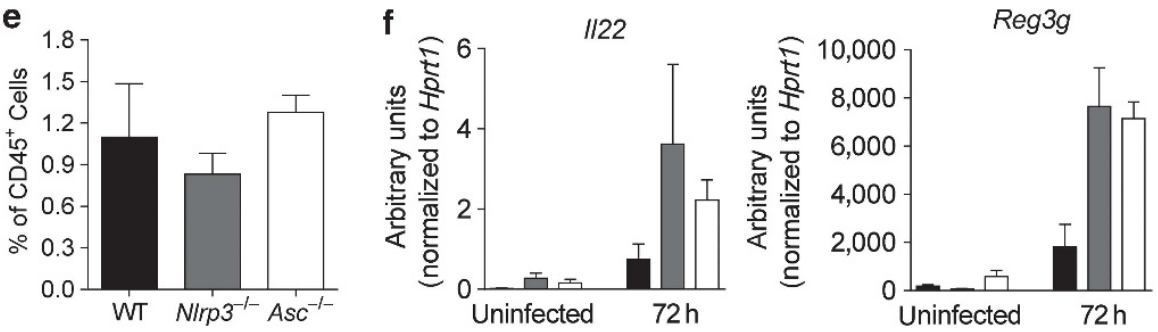
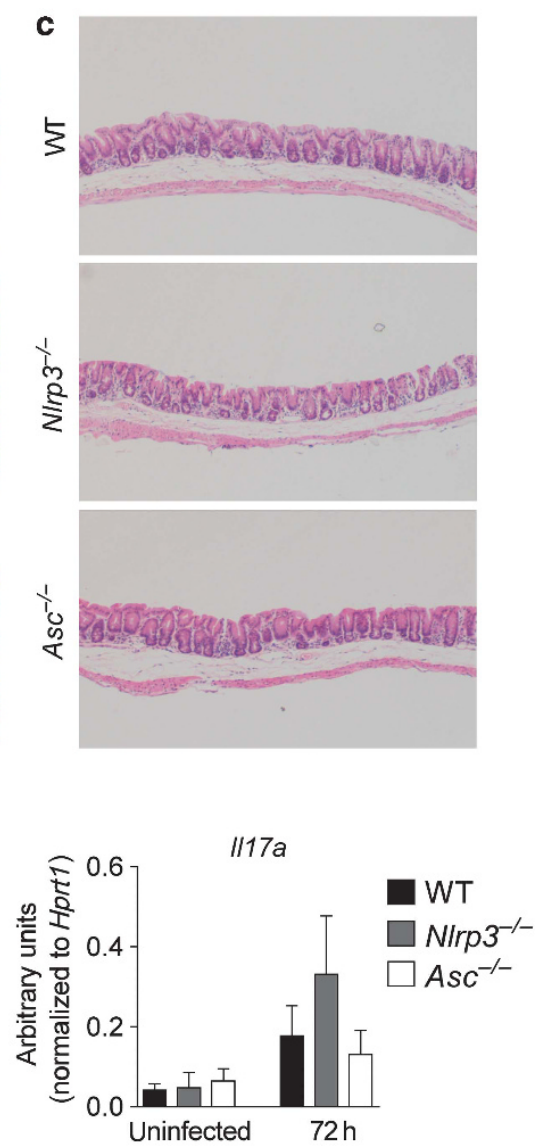

Figure 6 Nlrp3 and Asc activation mediates early control against Citrobacter rodentium. Cohorts of wild-type (WT), NIrp3 ${ }^{-/-}$, and Asc ${ }^{-/-}$mice were infected with $\sim 10^{9} \mathrm{C}$. rodentium and killed at $72 \mathrm{~h}$ after infection. (a) C. rodentium loads in the cecum at $72 \mathrm{~h}$ after infection $(n=17-20)$. (b) Cecal tissues from infected mice (72 h after infection) were stained for C. rodentium (red), E-cadherin (green), and 4', 6-diamidino-2-phenylindole (DAPI; blue). Left panel, bar $=20 \mu \mathrm{m}$, right panel, bar $=10 \mu \mathrm{m}$. (c) Representative photomicrographs depicting hematoxylin and eosin (H\&E) staining of $C$. rodentium infected ceca (original magnification $\times 50$ ). (d) Inflammation scores in the cecum at $72 \mathrm{~h}$ after infection $(n=6-11)$. (e) Frequency of CD45 ${ }^{+}$Lin $^{-}$Thy $1^{+}$ Sca ${ }^{+}$innate lymphoid cells (ILCs) of total CD $45^{+}$cells in the cecum at $72 \mathrm{~h}$ after infection $(n=4)$. (f) mRNA expression levels of $/ 122$, Reg3g, and $/ / 17 a$ at uninfected or $72 \mathrm{~h}$ after infection $(n=3)$. Data obtained from two independent experiments. Bar graphs (e, f) shown are means $( \pm \mathrm{s}$.e.m.). Horizontal bars (a, d) represent the medians. Statistical significance was determined by the Mann-Whitney test $\left({ }^{\star * \star} P<0.001 ;{ }^{* * \star \star} P<0.0001\right)$.

(Figure 6f), as well as comparable expression of several other AMPs (Supplementary Figure S4). Taken together with the bone marrow chimera results showing that the protection is mediated by nonhematopoietic cells, our data indicate that the early protection mediated by Nlrp3 and Asc activation is independent of the innate lymphoid cell/IL-22 axis. Furthermore, consistent with our previous findings at day 8 after infection (Figure 4a), we observed no difference in the levels of IL- $1 \alpha$, IL-1 $\beta$, IL-18, or active caspase-1 (p10) in the intestine of WT and Nlrp3 $3^{-1-}$ mice at $72 \mathrm{~h}$ after infection (Supplementary Figure S5), indicating that early Nlrp3 signaling in IEC protects from pathogenic infection independently of caspase- 1 activation.

To further investigate the mechanism through which Nlrp3 and Asc activation promotes early protection against C. rodentium infection, we performed genome-wide transcriptional profiling of cecal tissue at steady state and at $72 \mathrm{~h}$ after infection. We found that the expression of 26 genes were increased in WT ceca at $72 \mathrm{~h}$ after infection; however, the vast majority of these were also increased in $\mathrm{Nlrp3}^{-/-}$and $\mathrm{Asc}^{-/-}$ ceca (Supplementary Figure S6). In fact, upon infection only five genes were exclusively induced in WT mice but not in $N l r p 3^{-1-}$ or $A s c^{-1-}$ mice (Supplementary Figure S6). Of these five genes, the strongest candidate that could affect intestinal immune homeostasis was $\mathrm{Ccl} 5$ that encodes the chemokine CCL5, which has been implicated in recruitment of $\mathrm{T}$ cells, eosinophils, and basophils into inflammatory sites. ${ }^{27}$ However, subsequent quantitative PCR analyses were unable to validate the differential induction of $\mathrm{Ccl} 5$ between WT and $\mathrm{Nlrp3}^{-/-}$and $\mathrm{Asc}^{-/-}$mice (data not shown). Somewhat surprisingly, we identified 249 genes that were induced at significantly higher levels in Nlrp3 $3^{-1-}$ and/or $A s c^{-/-}$ceca at $72 \mathrm{~h}$ after infection, but not in WT ceca (Supplementary Figure S6). Approximately 50\% (124) of these genes were increased in both $\mathrm{Nlrp}^{-1-}$ and/or $A s c^{-1-}$ mice, suggesting that this expression profile may reflect responses to the higher bacterial levels present in Nlrp3- and Asc-deficient mice at $72 \mathrm{~h}$ after infection. Further analyses of the cecal gene expression profiles revealed that 39 probes, encompassing 33 different genes, were expressed at significantly higher levels in WT ceca compared 
with $\mathrm{Nlrp3}^{-/-}$and/or $\mathrm{Asc}^{-/-}$ceca (Supplementary Figure S7). Strikingly, the vast majority of these 33 genes were expressed at higher levels in WT mice both at steady state and at $72 \mathrm{~h}$ after infection (Supplementary Figure S7), suggesting that baseline differences in gene expression in the intestine of WT and Nlrp3- and Asc-deficient mice might contribute to protection from C. rodentium.

Collectively, these data show that the major protective effect of Nlrp3 and Asc activation in the intestinal epithelium is evident very early after infection with $C$. rodentium, is not associated with caspase- 1 activation, and does not seem to be mediated through the production of AMPs. Transcriptional profiling revealed differentially expressed genes between WT and Nlrp3- and Asc-deficient mice that were conserved between steady state and $72 \mathrm{~h}$ after infection. These observations raise the possibility that factors induced by constitutive Nlrp3 and Asc activation in the intestinal epithelium during steady-state conditions may limit early infection by mucosal pathogens.

\section{DISCUSSION}

Accumulating evidence suggests that innate immune recognition at mucosal surfaces particularly within the intestine is a crucial mediator of intestinal homeostasis. ${ }^{28}$ Apart from basal roles at steady state, pattern recognition receptor signaling confers protection against a multitude of enteric pathogens. ${ }^{29-31}$ Much of the work however has centered on the consequences of hematopoietic cell microbial detection, whereas nonhematopoietic cells such as IECs have been relatively understudied. As IECs are the first cells that enteric bacteria encounter, they are well poised to act as sentinels to limit invasion and alert the immune system to infection.

We first showed that mice lacking Nlrp3 exhibit exacerbated intestinal inflammation in response to infection with C. rodentium as compared with WT mice. This finding extends the previously described protective role of Nlrp3 in intestinal injury induced by chemical challenges. ${ }^{5-7}$ We also observed that $A s c^{-/-}$mice exhibited an even greater disease phenotype relative to the $N l r p 3^{-1-}$ mice, suggesting that other NLRs utilizing Asc may be involved in the protective response against C. rodentium.

To investigate which cellular compartment Nlrp3 and Asc activation provided protection against C. rodentium, we created irradiation bone marrow chimeras. These data revealed a major protective role for Nlrp3 and Asc activation in nonhematopoietic cells. Furthermore, using immunofluorescence microscopy, we observed that IECs ubiquitously expressed high levels of Asc, whereas very few cells in the lamina propria had detectable Asc, suggesting that IECs are the major cell type in which this protective Nlrp3 and Asc activation is occurring. C. rodentium colonizes the intestine by intimate attachment to IECs via a T3SS that delivers numerous virulence factors to the host. ${ }^{13}$ Collectively, these virulence factors enable C. rodentium to disrupt host processes, such as actin polymerization, water reabsorption, and epithelial tight junction maintenance, ultimately leading to intestinal inflammation and diarrhea. ${ }^{15}$
As IECs are the first cells to come into contact with C. rodentium, they are ideally positioned to detect $C$. rodentium and the outcomes of these virulent assaults. Consistent with this hypothesis, others have reported that MyD88 and NOD2 signaling within the nonhematopoietic compartment is important for optimal protection against C. rodentium infection. ${ }^{31-33}$ Our findings reinforce the concept that innate immune recognition at the epithelial barrier has a crucial function in the initiation of protective immunity ${ }^{34}$ and extend this paradigm to include activation of NLRs in IECs.

We next ascertained the mechanism through which Nlrp3Asc activation conferred protection. As $\mathrm{Asc}^{-1-}$ and Nlrp3 $3^{-/-}$ mice consistently harbored higher $C$. rodentium burdens than WT mice, we asked whether this could explain the exacerbated phenotype. As some enteric pathogens such as Salmonella typhimurium gain a selective advantage under inflammatory conditions, ${ }^{22,23}$ we could not be certain that higher C. rodentium levels were not merely a consequence of greater inflammation levels in $\mathrm{Asc}^{-1-}$ and $\mathrm{Nlrp3} 3^{-1-}$ mice. We thus measured the levels of $C$. rodentium in the tissue after $72 \mathrm{~h}$ of infection, before the onset of intestinal inflammation, and found that $N l r p 3^{-/-}$and $A s c^{-/-}$mice already harbored $\sim 1,000$-fold more tissue-adherent bacteria. Together, our findings show that signaling via Nlrp3 and Asc in nonhematopoietic cells early during the course of infection limits C. rodentium levels in the cecum and consequently dampens the ensuing intestinal inflammation.

Sensing of an infection at the epithelial barrier can have two foreseeable outcomes. First, activation of an effector antimicrobial response could directly limit the invading pathogen, such as the production of antimicrobial peptides. Second, recruitment and/or conditioning of other cells that subsequently clear the pathogen; for example, Nod2 sensing in stromal cells triggers the production of $\mathrm{Ccl} 2$, attracting inflammatory monocytes that promote $C$. rodentium clearance. $^{31}$ The first outcome would be predicted to be engaged with rapid kinetics early on in the response, whereas the second outcome might take relatively longer. Our result showing higher pathogen burdens after $72 \mathrm{~h}$ in $\mathrm{Nlrp3}^{-/-}$and $A s \mathrm{c}^{-/-}$ mice coupled with the nonhematopoietic phenotype suggests that an epithelial-intrinsic mechanism of action limits pathogen loads early on during infection, fitting with the predicted kinetics. Indeed, others have reported that $N l r p 3^{-/-}$cecal epithelial cells show a relatively impaired ability to kill $E$. coli compared with WT epithelial cells. ${ }^{35}$ Although none of the AMPs that we measured were deficient in $\mathrm{Asc}^{-/-}$or $N l r p 3^{-1-}$ mice, it is conceivable that an AMP might be regulated through a post-translational modification by an effector that is downstream of Nlrp3 and Asc activation. S100 proteins for instance are leaderless peptides and have been postulated to require caspase- 1 for their release; ${ }^{36}$ however, we did not find any defect in caspase- 1 activation in the intestines of $\mathrm{Nlrp3} 3^{-1-}$ mice.

Previous studies using the DSS colitis model reported a protective effect of Nlrp3 activation in nonhematopoietic cells and described a role for IL-18 in this protection. ${ }^{5,6}$ In addition, a 
recent study reported that Nlrp3 and caspase-1 played a protective role during infection with $C$. rodentium and ascribed this protective effect to the production of the caspase-1processed cytokines IL-1 $\beta$ and IL-18. ${ }^{37}$ However, this study focused only on the latter stages of $C$. rodentium infection, from day 14 after infection onwards, and did not identify the cell types responsible for Nlrp3-mediated protection. ${ }^{37}$ Our work has revealed that activation of Nlrp3 and Asc in intestinal epithelial cells acts very early in the course of infection to restrict bacterial replication and spread that in turn limits the severity of subsequent intestinal infection. During this early phase of infection, we did not find any induction of IL-1 $\beta$ and we observed equivalent IL-18 production between $\mathrm{Nlrp3}^{-/-}$and WT mice, whereas IL-18 production was completely abrogated in $\mathrm{Asc}^{-/-}$mice. Consistent with this expression pattern, we found that $N l r p 3^{-/-}$mice expressed equivalent levels of activated caspase-1 (p10) relative to WT mice after C. rodentium infection, whereas caspase- 1 cleavage was greatly diminished in $\mathrm{Asc}^{-/-}$mice. The simplest explanation for these observations is that Nlrp3 activation protects against C. rodentium-driven disease independently of caspase- 1 activation. However, it is possible that the intact caspase- 1 activation observed in the absence of Nlrp3 was because of compensatory activation of alternative inflammasome-forming NLRs, although this was unable to rescue the exacerbated disease phenotype. Thus, although we cannot definitively exclude a potential role for caspase-1 in Nlrp3-mediated protection, such a hypothesis would imply that discrete, nonredundant effector pathways are triggered following caspase- 1 activation by distinct inflammasomes. Furthermore, although Asc- and caspase-1-dependent IL-18 production might explain the difference in disease severity between Nlrp $3^{-/-}$and $A s c^{-/-}$mice, it cannot account for the major susceptibility phenotype observed in $\mathrm{Asc}^{-/-}$mice compared with WT mice. This also implies that other inflammasomeforming NLRs may make a minor contribution to protection from $C$. rodentium through the production of IL-18. Consistent with a relatively minor role for IL-18 in immunity against C. rodentium, others have also reported that $I l 18^{-/-}$mice display a mild phenotype in this model. ${ }^{38}$ In addition, we observed that $I l 18 \mathrm{rl}^{-1-}$ mice developed an intermediate phenotype after infection with $C$. rodentium; they harbored greater $C$. rodentium levels in their ceca and spleens but did not develop significantly greater intestinal inflammation compared with WT mice. Thus, the major protective effect of epithelial Nlrp3 activation appears to be independent of caspase-1. This finding has important implications for our understanding of NLR activation in distinct cell types, as it suggests that the Nlrp3-Asc signaling circuits that have been elucidated through studies in hematopoietic cells may not be similarly hard-wired in other cell types. When taken together with the findings of Liu et al. ${ }^{37}$ our results suggest that there may be two phases of Nlrp3/Asc activation that contribute to protection from $C$. rodentium: the very early IEC-intrinsic innate pathway described in our study, and a later circuit, most likely in myeloid cells, leading to classical caspase-1 inflammasome activation and the secretion of IL-1 $\beta$ and IL-18. ${ }^{37}$ Evidence of the important contribution of the latter pathway is provided by the reported susceptibility phenotypes of Casp1 $1^{-/-}$and Illr $^{-1-}$ after infection with C. rodentium..$^{37,38}$

The effector pathways triggered following Nlrp3 activation in nonhematopoietic cells have not been well studied, but additional proteins linked to NLR activation, such as HMGB1 released by means of unconventional protein secretion, might also contribute to protection against $C$. rodentium and will be investigated in the future. ${ }^{17,39,40}$ Furthermore, it was recently shown that activation of the related NLR, Nlrc4, triggered caspase-1-mediated release of eicosanoids, such as leukotrienes and prostaglandins, independently of IL- $1 \beta$ or IL-18 secretion, highlighting that selective release of distinct classes of inflammatory mediators can occur in myeloid cells after NLR activation. ${ }^{41}$ Another consequence of inflammasome activation in myeloid cells is an inflammatory form of cell death known as pyroptosis. ${ }^{2,3}$ An attractive hypothesis is that early Nlrp3 activation in IECs might induce pyroptosis in IECs, and this "altruistic" cell death could limit the replicative niche for C. rodentium. However, we found very few numbers of TUNEL $^{+}$IECs in the intestines of $\mathrm{Nlrp}^{-/-}, \mathrm{Asc}^{-/-}$, and WT mice after infection with C. rodentium (data not shown). Furthermore, mice lacking caspase-11, a key upstream mediator of pyroptosis, displayed similar susceptibility to C. rodentium infection compared with WT mice. ${ }^{42}$ Thus, the potential role of pyroptosis in protection against C. rodentium infection remains to be resolved.

An open question remains as to how precisely $C$. rodentium triggers Nlrp3 and Asc activation. Several studies have shown that $C$. rodentium, upon injection of effector proteins into epithelial cells, destabilize host cell mitochondria and cause apoptosis. ${ }^{15}$ Recent studies of the mechanism of Nlrp3 activation have uncovered a role for mitochondrial dysfunction in the triggering of inflammasome activation, ${ }^{43,44}$ suggesting that T3SS-delivered effector proteins could potentiate Nlrp3 activation within IECs. In contrast, a recent study showed that the $C$. rodentium T3SS was dispensable for activating the inflammasome in BM-derived macrophages in vitro. ${ }^{37}$ Future work will address this question using a panel of virulence factor-deficient $C$. rodentium mutants in vivo.

Overall, our study has identified an early protective circuit in the intestinal epithelium driven by signaling though Nlrp3 and Asc that provides protection against bacterial-driven intestinal inflammation. This may constitute a key function through which Nlrp3 and Asc help maintain intestinal homeostasis and could represent the dysregulated pathway in Crohn's disease patients with single-nucleotide polymorphisms in NLRP3.

\section{METHODS}

Ethics statement. Animal experiments were conducted in accordance with the UK Scientific Procedures Act of 1986 under a Project License authorized by the UK Home Office Animal Procedures Committee 
and approved by the Sir William Dunn School of Pathology Local Ethical Review Committee.

Mice. WT C57BL/6 (B6), 129SvEv, B6.Asc ${ }^{-/-}$, B6.Ill8r1 $1^{-/-}$ (Jackson Laboratory, Bar Harbor, ME), and B6.Nlrp3 $3^{-/-}$mice were bred and maintained under specific pathogen-free conditions in accredited animal facilities at the University of Oxford (Oxford, UK). Mice were older than 6 weeks of age when used and age and sex matched. Experimental cohorts were co-housed for at least 2 weeks before infection with $C$. rodentium. During $C$. rodentium infection experiments, WT, Nlrp3 ${ }^{-/-}$, and $A s c^{-1-}$ cohorts were either cohoused throughout the entire infection period or were re-segregated into their respective genotypes and housed separately after infection. We obtained similar results irrespective of whether co-housing was maintained or discontinued after infection with C. rodentium.

Quantitative PCR. Total RNA was isolated using RNeasy Mini Kit (Qiagen, Hilden, Germany). RNA was reverse transcribed using Superscript III reverse transcriptase (Invitrogen, Carlsbad, CA) and oligo-dT primers. Gene expression was assessed using primer and probes from Applied Biosystems (Foster City, CA) TaqMan Gene Expression Assay on a Chromo4 detection system (MJ Research, Waltham, MA). Expression levels were normalized to Hprt1 and calculations were made using the $2^{-\Delta \mathrm{Ct}}$ method. ${ }^{45}$

Bacterial infection. A single C. rodentium colony was transferred to nalidixic acid-supplemented Luria-Bertani broth and grown overnight to saturation. The next day, the culture was diluted to an optical density of 0.05 and grown to log phase before harvest by centrifugation and resuspension in phosphate-buffered saline. Mice were orally gavaged with $200 \mu \mathrm{l}$ of phosphate-buffered saline containing $\sim 10^{9}$ C. rodentium (nalidixic acid resistant) (ICC169). Following infection, mice were weighed every day and culled if weight loss exceeded $20 \%$ of starting weight.

Colony counts of $\boldsymbol{C}$. rodentium. To measure the $C$. rodentium load in infected mice, tissues were weighed and then homogenized in $600 \mu \mathrm{l}$ of phosphate-buffered saline. Serial dilutions of tissue lysates were plated on nalidixic acid (Sigma-Aldrich, St Louis, MO; final concentration $50 \mu \mathrm{g} \mathrm{ml}^{-1}$ ) agar plates and then incubated at $37^{\circ} \mathrm{C}$ overnight before counting colonies. The numbers of colonies were normalized to the weight of the tissue (CFU per g).

Total protein extracts and immunoblot analysis. Total protein extracts were prepared by homogenizing snap frozen cecal tissue in RIPA buffer containing a protease inhibitor cocktail (Roche, Basel, Switzerland). Protein levels were equalized by the Lowry assay (Bio-Rad Laboratories, Hercules, CA), resolved by sodium dodecyl sulfate-polyacrylamide gel electrophoresis, and then analyzed with anti-caspase-1 p10 (Santa Cruz Biotechnology, Dallas, TX; sc-514), anti-IL-18 (Abcam, Cambridge, UK; ab71495), and anti-Tubulin (Santa Cruz sc5286). Visualization and imaging were then carried out using ECL solution (Pierce, Rockford, IL).

Irradiation BM chimeras. BM was isolated from the tibias and fibulas of WT C57BL/6, Nlrp $3^{-/-}$, or Asc ${ }^{-1-}$ mice, and $10 \times 10^{6}$ cells were injected intravenously into $\gamma$-irradiated 11 Gy $(2 \times 550 \mathrm{rad}$, given $4 \mathrm{~h}$ apart) recipient mice and left for at least 6 weeks for reconstitution.

Organ explant culture. To measure cytokine production, tissues were weighed, washed in antibiotic supplemented phosphate-buffered saline, and then incubated overnight in complete RPMI at $37^{\circ} \mathrm{C}$. Supernatants were harvested and the levels of IL-1 $\alpha$ (eBioscience, San Diego, CA; 88-5019-22), IL-1 $\beta$ (coating antibody: eBioscience; 14-7012-85; detection antibody: eBioscience; 13-7112-85; standard: PeproTech, Rocky Hill, NJ; 211-11b), and IL-18 (eBioscience; BMS618/2TEN) measured by enzyme-linked immunosorbent assay and interferon- $\gamma$, IL-17A, IL-22, and tumor necrosis factor- $\alpha$ were quantified using Flow Cytomix Cytokine Bead Assay (Bender MedSystems, Vienna, Austria). ${ }^{46}$
Assessment of intestinal inflammation. Mice were killed at the indicated time points during the course of infection whereupon tissue sections were cut and fixed in buffered $10 \%$ formalin. Sections were then cut and stained with hematoxylin and eosin. Sections of cecum and distal colon were then blinded and scored by two researchers. In summary, five categories were considered (each scored 0-3): epithelial hyperplasia/damage and goblet cell depletion; leukocyte infiltration in lamina propria; submucosal inflammation and edema; area of tissue affected; and markers of severe inflammation such as bleeding, crypt abscesses, and necrosis/ulceration. The sums of these five categories are presented in the figures (scored 0-15).

Isolation of leukocyte subpopulations and fluorescence-activated cell sorting. Cell suspensions from spleen, and the lamina propria were prepared as described previously. ${ }^{47}$ Cells were washed and incubated with anti-Fc receptor (anti CD16/32 from eBioscience, San Diego, CA) at $4{ }^{\circ} \mathrm{C}$. Next, cells were washed and stained for CD11b and $\mathrm{Gr1}$, before being fixed overnight at $4^{\circ} \mathrm{C}$. Finally, cells were washed twice and acquired with a Cyan (Dako, Glostrup, Denmark) and analysis performed using FlowJo (TreeStar, Ashland, OR) software.

Gene expression analysis. Cecal RNA was extracted using the RiboPure kit (Ambion, Austin, TX) and whole genome expression was profiled using Illumina (San Diego, CA) Single-Color Mouse WG-6_V2_0_R0_11278593 BeadChip with direct hybridization assay. Biotinylated cRNA was prepared using the Illumina TotalPrep-96 RNA Amplification Kit (\#4393543 Ambion). Fluorescence emissions by Cy3 were imaged using iScan system (Illumina), and data was generated using the GenomaStudio 2011 software (Illumina). Data was imported to GeneSpring GX 12 (Agilent Technologies, Santa Clara, CA) for analysis.

Fluorescence microscopy. Paraformaldehyde-fixed tissue sections were deparaffinized and antigen retrieval was performed in $0.01 \mathrm{M}$ sodium citrate buffer. Slides were blocked with normal goat serum and stained with rabbit anti-C. rodentium antiserum, ${ }^{48}$ mouse anti-E-cadherin (BD Bioscience, San Jose, CA), and rabbit anti-Asc (Santa Cruz; sc 22514-R) followed by an AF555-conjugated goat-antirabbit and an AF488-conjugated goat-anti-mouse secondary antibody (Invitrogen). Slides were mounted in DAPI-containing Vectashield (Vector Laboratories, Burlingame, CA) and then visualized using an FV1000 confocal microscope (Olympus, Tokyo, Japan).

Statistics. Statistical significance was determined by two-way analysis of variance with Bonferroni post-tests for weight curves. All other statistical significance was determined either by nonparametric Mann-Whitney test or by paired $t$-tests. Differences were considered statistically significant when $P<0.05$.

SUPPLEMENTARY MATERIAL is linked to the online version of the paper at http://www.nature.com/mi

\section{ACKNOWLEDGMENTS}

We thank V.M. Dixit (Genentech, USA) for providing NIrp3 ${ }^{-1-}$ and $\mathrm{Asc}^{-1-}$ mice; R. Stillion for histology; PSB staff for animal maintenance; High Throughput Genomics, The Wellcome Trust Centre for Human Genetics for running the microarray; and $\mathrm{C}$. Schiering for advice on bioinformatic analysis. This work was supported by grants from the Wellcome Trust (to K.J.M. and G.F.), a MSD-Norman Heatley Studentship (to G.X.S.-Z.), the Clarendon Fund (to G.X.S.-Z. and N.S.), and an EMBO fellowship (to J.P.).

\section{DISCLOSURE}

The authors declared no conflict of interest.

c 2014 Society for Mucosal Immunology

\section{REFERENCES}

1. Schroder, K. \& Tschopp, J. The inflammasomes. Cell 140, 821-832 (2010). 
2. Miao, E.A. et al. Caspase-1-induced pyroptosis is an innate immune effector mechanism against intracellular bacteria. Nat. Immunol. 11, 1136-1142 (2010).

3. Kayagaki, N. et al. Non-canonical inflammasome activation targets caspase-11. Nature 479, 117-121 (2011).

4. Villani, A.C. et al. Common variants in the NLRP3 region contribute to Crohn's disease susceptibility. Nat. Genet. 41, 71-76 (2009).

5. Zaki, M.H. et al. The NLRP3 inflammasome protects against loss of epithelial integrity and mortality during experimental colitis. Immunity 32 379-391 (2010)

6. Dupaul-Chicoine, J. et al. Control of intestinal homeostasis, colitis, and colitis-associated colorectal cancer by the inflammatory caspases. Immunity 32, 367-378 (2010).

7. Allen, I.C. et al. The NLRP3 inflammasome functions as a negative regulator of tumorigenesis during colitis-associated cancer. J. Exp. Med. 207, 1045-1056 (2010).

8. Bauer, C. et al. Colitis induced in mice with dextran sulfate sodium (DSS) is mediated by the NLRP3 inflammasome. Gut 59, 1192-1199 (2010).

9. Bauer, C. et al. The ICE inhibitor pralnacasan prevents DSS-induced colitis in C57BL/6 mice and suppresses IP-10 mRNA but not TNF-alpha mRNA expression. Dig. Dis. Sci. 52, 1642-1652 (2007).

10. Siegmund, B. et al. IL-1 beta-converting enzyme (caspase-1) in intestinal inflammation. Proc. Natl. Acad. Sci. USA 98, 13249-13254 (2001).

11. Sivakumar, P.V et al. Interleukin 18 is a primary mediator of the inflammation associated with dextran sulphate sodium induced colitis: blocking interleukin 18 attenuates intestinal damage. Gut 50, 812-820 (2002).

12. Luperchio, S.A. \& Schauer, D.B. Molecular pathogenesis of Citrobacter rodentium and transmissible murine colonic hyperplasia. Microbes. Infect. 3, 333-340 (2001).

13. Mundy, R. et al. Citrobacter rodentium of mice and man. Cell. Microbiol. 7 1697-1706 (2005)

14. Elinav, E. et al. NLRP6 inflammasome regulates colonic microbial ecology and risk for colitis. Cell 145, 745-757 (2011).

15. Garmendia, J. et al. Enteropathogenic and enterohemorrhagic Escherichia coli infections: translocation, translocation, translocation. Infect. Immun. 73, 2573-2585 (2005).

16. Martinon, F. et al. The inflammasome: a molecular platform triggering activation of inflammatory caspases and processing of prolL-beta. Mol. Cell. 10, 417-426 (2002).

17. Gross, O. et al. Inflammasome activators induce interleukin-1alpha secretion via distinct pathways with differential requirement for the protease function of caspase-1. Immunity 36, 388-400 (2012).

18. Maaser, C. et al. Clearance of Citrobacter rodentium requires $B$ cells but not secretory immunoglobulin A (IgA) or IgM antibodies. Infect. Immun. 72, 3315-3324 (2004).

19. Vallance, B.A. et al. Mice lacking $T$ and B lymphocytes develop transient colitis and crypt hyperplasia yet suffer impaired bacterial clearance during Citrobacter rodentium infection. Infect. Immun. 70 2070-2081 (2002).

20. Torchinsky, M.B. et al. Innate immune recognition of infected apoptotic cells directs $T(H) 17$ cell differentiation. Nature 458, 78-82 (2009).

21. Mangan, P.R. et al. Transforming growth factor-beta induces development of the $T(H) 17$ lineage. Nature 441, 231-234 (2006).

22. Stecher, B. et al. Salmonella enterica serovar typhimurium exploits inflammation to compete with the intestinal microbiota. PLoS Biol. 5, 2177-2189 (2007)

23. Winter, S.E. et al. Gut inflammation provides a respiratory electron acceptor for Salmonella. Nature 467, 426-429 (2010).

24. Sonnenberg, G.F. et al. CD4(+) lymphoid tissue-inducer cells promote innate immunity in the gut. Immunity 34, 122-134 (2011).
25. Tumanov, A.V. et al. Lymphotoxin controls the IL-22 protection pathway in gut innate lymphoid cells during mucosal pathogen challenge. Cell. Host. Microbe. 10, 44-53 (2011).

26. Zheng, Y. et al. Interleukin-22 mediates early host defense against attaching and effacing bacterial pathogens. Nat. Med. 14, 282-289 (2008).

27. D'Ambrosio, D. et al. Chemokine receptors in inflammation: an overview. J. Immunol. Methods 273, 3-13 (2003).

28. Abreu, M.T. Toll-like receptor signalling in the intestinal epithelium: how bacterial recognition shapes intestinal function. Nat. Rev. Immunol. 10, 131-144 (2010).

29. Muller, A.J. et al. The S. Typhimurium effector SopE induces caspase-1 activation in stromal cells to initiate gut inflammation. Cell. Host. Microbe. 6 , 125-136 (2009).

30. Jarchum, I. et al. Critical role for MyD88-mediated neutrophil recruitment during Clostridium difficile colitis. Infect. Immun. 80, 2989-2996 (2012).

31. Kim, Y.G. et al. The Nod2 sensor promotes intestinal pathogen eradication via the chemokine CCL2-dependent recruitment of inflammatory monocytes. Immunity 34, 769-780 (2011).

32. Gibson, D.L. et al. MyD88 signalling plays a critical role in host defence by controlling pathogen burden and promoting epithelial cell homeostasis during Citrobacter rodentium-induced colitis. Cell. Microbiol. 10, 618-631 (2008).

33. Lebeis, S.L. et al. TLR signaling mediated by MyD88 is required for a protective innate immune response by neutrophils to Citrobacter rodentium. J. Immunol. 179, 566-577 (2007).

34. Fritz, J.H. et al. Innate immune recognition at the epithelial barrier drives adaptive immunity: APCs take the back seat. Trends. Immunol. 29, 41-49 (2008).

35. Hirota, S.A. et al. NLRP3 inflammasome plays a key role in the regulation of intestinal homeostasis. Inflamm. Bowel. Dis. 17, 1359-1372 (2011).

36. Lamkanfi, M. Emerging inflammasome effector mechanisms. Nat. Rev. Immunol. 11, 213-220 (2011)

37. Liu, Z. et al. Role of inflammasomes in host defense against Citrobacter rodentium infection. J. Biol. Chem. 287, 16955-16964 (2012).

38. Lebeis, S.L. et al. Interleukin-1 receptor signaling protects mice from lethal intestinal damage caused by the attaching and effacing pathogen Citrobacter rodentium. Infect. Immun. 77, 604-614 (2009).

39. Keller, M. et al. Active caspase- 1 is a regulator of unconventional protein secretion. Cell 132, 818-831 (2008).

40. Lamkanfi, M. et al. Inflammasome-dependent release of the alarmin HMGB1 in endotoxemia. J. Immunol. 185, 4385-4392 (2010).

41. von Moltke, J. et al. Rapid induction of inflammatory lipid mediators by the inflammasome in vivo. Nature 490, 107-111 (2012).

42. Gurung, P. et al. Toll or interleukin-1 receptor (TIR) domain-containing adaptor inducing interferon- $\beta$ (TRIF)-mediated caspase-11 protease production integrates Toll-like receptor 4 (TLR4) protein- and Nlrp3 inflammasome-mediated host defense against enteropathogens. J. Biol. Chem. 287, 34474-34483 (2012).

43. Zhou, R. et al. A role for mitochondria in NLRP3 inflammasome activation. Nature 469, 221-225 (2011).

44. Shimada, K. et al. Oxidized mitochondrial DNA activates the NLRP3 inflammasome during apoptosis. Immunity 36, 401-414 (2012).

45. Boulard, O. et al. TLR2-independent induction and regulation of chronic intestinal inflammation. Eur. J. Immunol. 40, 516-524 (2010).

46. Ahern, P.P. et al. Interleukin-23 drives intestinal inflammation through direct activity on T cells. Immunity 33, 279-288 (2010).

47. Uhlig, H.H. et al. Characterization of Foxp3 + CD4 + CD25 + and IL-10secreting CD4 + CD25 + T cells during cure of colitis. J. Immunol. 177, 5852-5860 (2006).

48. Mundy, R. et al. Comparison of colonization dynamics and pathology of mice infected with enteropathogenic Escherichia coli, enterohaemorrhagic E. coli and Citrobacter rodentium. FEMS Microbiol. Lett. 265, 126-132 (2006). 